\title{
ANATOMÍA ENDOSCÓPICA DE LA UNIDAD ESTRUCTURAL FISURA ORBITARIA INFERIOR - MÚSCULO DE MÜLLER EN LA JUNCIÓN ÓRBITO SELAR MEDIAL Y SU IMPORTANCIA QUIRÚRGICA.
}

\author{
ENDOSCOPIC ANATOMY OF THE INFERIOR ORBITAL FISSURE-MÜLLER'S MUSCLE STRUCTURAL \\ UNIT AT THE MEDIAL SELLAR ORBITAL JUNCTION AND ITS SURGICAL RELEVANCE
}

Juan C. De Battista', Carlos F. Buonanotte, Gustavo A. Foa Torres, Jeffrey T. Keller.

\section{Resumen:}

Antecedentes: Las enfermedades que afectan la órbita representan un desafío quirúrgico, en particular las que comprometen el ápex orbitario. Una vía óptima de acceso quirúrgico proporciona la mejor exposición permitiendo identificar ciertas estructuras anatómicas claves llamadas reparos anatómicos. Objetivo: Describir la anatomía endoscópica de la unidad estructural Fisura Orbitaria Inferior / Músculo de Müller a nivel del ápex orbitario generando así un nuevo reparo anatómico endoscópico. Material y método: Análisis descriptivo óseo de la fisura orbitaria inferior (FOI) en cráneos secos, disección y estudio bajo técnica endoscópica de 6 cabezas fijadas en formol y coloreadas; finalmente se tomaron distancias y ángulos a forámenes relacionados con el ápex orbitario a 10 cráneos secos. El análisis estadístico se realizó con el programa estadístico SPSS 17,0 (SPSS, Inc., Chicago, IL). Resultado: En todas las disecciones endoscópicas se pudo identificar la unidad fisura orbitaria inferior-músculo de Müller y también verificar su íntima relación con el ápex orbitario. Morfométricamente el foramen óptico y el foramen redondo mayor están a una distancia promedio de $65.19 \mathrm{~mm}$ y $60.16 \mathrm{~mm}$ respectivamente. Los ángulos promedio del FO fue de 13.32 grados y del FRM de19.31 grados. Hallamos correlación significativa entre CO. y el FRM sólo en el hemicráneo izquierdo, (Tau b de Kendall 0.69, $P=0.006$ ). No se encontraron diferencias anatómicas (o morfológicas) significativas entre lados. Conclusión: bajo técnica endoscópica la unidad Fisura Orbitaria Inferior-Músculo de Müller (FOI-MM) es un reparo anatómico constante, útil y seguro que permite el reconocimiento del ápex orbitario y sus áreas contiguas.

Palabras clave: base de cráneo; anatomía; endoscopía; órbita

\section{Abstract:}

Background: Diseases of the orbit represent a surgical challenge, particularly those compromising the orbital apex. Optimal surgical access should provide the best possible exposure, allowing to identify certain key anatomical structures, called landmarks. Objective: Describe the endoscopic anatomy of the structural unit formed by the Inferior Orbital Fissure (IOF) and the Müller's muscle (MM) at the orbital apex (OA), thus generating a new endoscopic anatomical landmark. Materials and methods: A bone-descriptive analysis of the IOF in dry craniums, was followed by dissection and endoscopic study of six heads (twelve sides), colored and fixed in formaldehyde. In ten dry craniums (twenty sides), distances and angles of OA foramina were measured (optic foramen [OF] and foramen rotundum [FR]). Statistical analysis was performed with SPSS 17.0 statistical software (SPSS, Inc. Chicago, IL). Results: The structural unit IOFMM was identified in all endoscopic dissections, verifying its intimate relationship with the OA. From the morpho-metric standpoint, OF and FR were found at an average distance of $65.19 \mathrm{~mm}$ and $60.16 \mathrm{~mm}$, respectively. The average angle of the OF was 13.32 degrees, whereas the one for FR was 19.31 degrees. We found a significant correlation between OF and FR only on the left side (left hemi-crane) (Kendall Tau $b$ $0.69, \mathrm{p}=0.006$ ). There were no anatomical or morphological differences between both sides. Conclusion: The unit IOF-MM is a constant anatomical landmark, useful and safe under endoscopic technique, which allows the recognition of the $\mathrm{OA}$ and its contiguous areas.

Keywords: skull base; anatomy; endoscopy; orbit

\footnotetext{
${ }^{1}$ Médico neurocirujano del departamento de neurocirugía del Hospital Privado Centro Médico Universitario de Córdoba y doctorando del Doctorado en Medicina y Cirugía de la FCM de la UNC. Doctor Profesor Carlos F. Buonanotte, Titular de la Cátedra de neurología de la facultad de ciencias médicas (FCM) de la UNC. Jefe de servicio de neurología del Hospital Nacional de Clínicas de Córdoba. Doctor Profesor Gustavo A. Foa Torres, Titular de la Cátedra de Diagnóstico por Imágenes de la UNC. Jefe de neruoradiología diagnóstica y terapéutica del Instituto Oulton de Córdoba. Doctor Profesor Jeffrey T. Keller, Co-director de la Tesis de De Battista, Jefe del Departamento de neurocirugía de la Universidad de Cincinnati (Instituto de neurociencia, Cincinnati, Ohio, EEUU). Doctor Profesor Cesar I. Aranega director de la tesis doctoral de la UNC.
} 


\section{Introducción}

Las enfermedades que afectan a la órbita representan un desafío quirúrgico, en particular las que comprometen el ápex orbitario. La vía óptima de acceso quirúrgico es aquella que proporciona la mejor exposición posible permitiendo identificar ciertas estructuras anatómicas claves llamadas reparos anatómicos. Las mismas deben ser constantes y en lo posible analizables en los estudios preoperatorios. De esta forma los reparos anatómicos nos brindan la posibilidad de generar un plan peri-operatorio (pre e intraoperatorio) y por lo tanto mayor seguridad durante el acto quirúrgico. A su vez, como sabemos, la elección óptima del abordaje quirúrgico también dependerá del proceso patológico en cuestión ${ }^{(1,2,3)}$. Los abordajes quirúrgicos tradicionales al ápex orbitario incluyen, entre otros, orbitotomías laterales y craneotomías con componente orbitario, ambos procedimientos muy invasivos y con significativa morbilidad si los comparamos a los empleados en los abordajes quirúrgicos endoscópicos ${ }^{(4,5,6,7,1,8)}$.

Además de los clásicos, otros tipos de abordajes posibles a esta región son los endoscópicos tansetmoidales y transmaxilares. $(9,10,11,12)$. En los procesos patológicos localizados en la cara medial e inferior del ápex orbitario, los abordajes laterales 0 craneales (no endoscópicos) o clásicos pueden ser vías de difícil acceso a esta profunda región de la base de cráneo. A diferencia de estos, la vía por corredores endoscópicos endonasales 0 transmaxilares generan una trayectoria de trabajo directa al target y la visualización de estas caras orbitarias se obtiene con mayor facilidad $y$ amplitud $^{(10,13)}$.

Sin embargo, es lento el reemplazo de los abordajes neuroquirúrgicos tradicionales a los endoscópicos e influye más de un factor en la falta de uso cotidiano del endoscopio para las lesiones del ápex orbitario. En efecto para los abordajes de esta profunda y compleja región se necesita conocer de modo preciso lo que denominamos anatomía endoscópica El entrenamiento en técnicas endoscópicas (endoneurocirujanos) y el estudio minucioso de las estructuras y anatomía de la base craneal son indispensables para interpretar las imágenes que llegarán al monitor invertidas y distorsionadas por la lente de la cámara endoscópica. A su vez este estudio anatómico es complementario al obtenido tradicionalmente, proporcionándonos una información invalorable de la anatomía en sus 360 grados
Pasada la primera década del actual siglo, la ausencia de trabajos suficientes y de bibliografía específica que brinde una descripción más profunda y exhaustiva de estas regiones nos llevó a estudiar con más detalle la fisura orbitaria inferior (FOI), lo que luego se convirtió en objeto central la tesis doctoral (14,15y16). A través del estudio minucioso, del relevamiento de imágenes y de la toma de medidas de esta área, buscamos establecer y señalizar los reparos anatómicos ineludibles en la cirugía endoscópica, poniendo de relieve que esta región está íntimamente relacionada con el ápex orbitario y explicando el modo en que entra en contacto con la fisura orbitaria superior $^{(17,18,19,20)}$.

Por tanto el objetivo de nuestro trabajo es demostrar la relación de unidad entre la fisura orbitaria inferior (reparo óseo clave) y el músculo de Müller (otro reparo visual endoscópico necesario) a nivel del ápex orbitario, más precisamente en la unión órbito-selar medial, lo que denominamos Junción órbito-selar medial (JOSM).

\section{Materiales y Métodos}

En primera instancia se realizó un análisis descriptivo óseo de la fisura orbitaria inferior (FOI) en 50 cráneos secos (100 lados) más precisamente su segmento posteromedial. Luego se llevó a cabo la disección y estudio de 6 cabezas (12 lados) fijadas en formol. Se rellenaron sus vasos con silicona coloreada con pintura roja (sistema arterial) y azul (sistema venoso) para mejorar la comprensión anatómica de esta región. Las cabezas estudiadas anatómicamente en este trabajo se disecaron empleando un endoscopio rígido de $4 \mathrm{~mm}$ de diámetro, $14018 \mathrm{~cm}$ de largo, y lentes de $0^{\circ}$ y $30^{\circ}$ (Karl Storz o Aesculap). El endoscopio fue acoplado a una fuente de luz y a una videocámara conectada a un monitor. Las imágenes y videos fueron registrados por un sistema digital (Torre de endoscopia Strayker, Kalamazoo, MI).

En la estación de trabajo Brain Lab Vector Vision Navigation System del servicio de neurocirugía de la Universidad de Cincinnati (EEUU) se tomaron diversas medidas en 10 de los cráneos secos (es decir, 20 lados) a partir de su digitalización luego de realizarles una tomografía computada (TC). Las distancias fueron desde la espina nasal del hueso maxilar inferior hasta el canal o foramen óptico y foramen redondo mayor. Además se definió un ángulo lateral desde la línea media hacia la derecha o izquierda de cada orificio estudiado. Estos ángulos se tomaron entre el plano medial y constante 
(creado al unir una línea virtual entre la espina nasal y el pico o rostrum del esfenoides), y el plano dado por la línea entre la espina nasal y cada uno de los forámenes (Figura 1). El objetivo consistió en conocer el ángulo de dichos forámenes con respecto al plano sagital medial, utilizado frecuentemente en los abordajes endoscópicos dirigidos a la región selarhipofisaria.

Las mediciones obtenidas fueron trabajadas a partir de un análisis estadístico: las variables continuas se expresan como media y desviación estándar o como mediana y rango intercuartil en función de su homogeneidad. Las variables continuas se compararon con la prueba de la t de Student o la prueba de Mann-Whitney de acuerdo a su homogeneidad. Un valor de probabilidad $<0,05$ se consideró significativo. El análisis estadístico se realizó con el programa estadístico SPSS 17,0 (SPSS, Inc., Chicago, IL).

\section{Resultados}

1-Morfométrico: El foramen o canal óptico, (CO por donde pasan el nervio óptico y la arteria oftálmica) se encuentra a una distancia $65.19 \mathrm{~mm}$ (Derecho: 65.24; Izquierdo: 65.14; P: 0.86) de la espina nasal del hueso maxilar superior. El ángulo lateral promedio es de 13.32 grados (Derecho: 13.3; Izquierdo: 13.35; P: 0.95).

El Foramen redondo mayor (FRM por donde pasa el nervio maxilar superior, segunda rama del nervio trigémino): distancia de la espina nasal del hueso maxilar superior $60.16 \mathrm{~mm}$ (Derecho: 59.74; Izquierdo: 60.58; P: 0.33). El ángulo lateral promedio es de 19.31 grados (Derecho: 19.21; Izquierdo: 19.41; P: 0.82). Hallamos una correlación significativa entre las distancias del CO y el FRM sólo del lado izquierdo (hemicráneo izquierdo) (Tau b de Kendall 0.69; $P=0.006$ ). La diferencia en la distancia y ángulo entre el foramen óptico y el redondo mayor es respectivamente de $5.03 \mathrm{~mm}$ y 6 grados. En otras palabras, el ángulo nos evidencia que el FRM está en posición de 6 grados más lateral que el óptico. A su vez este último, al ser más craneal (es decir, más alto), su distancia puerta de entrada-target (forámenes) es $5 \mathrm{~mm}$ mayor.

2-Descriptivo óseo: Como resultado de nuestro estudio e investigación precisamos y definimos la FOI o fisura esfeno-maxilar como un estrecho espacio entre el ala mayor del esfenoide y el seno maxilar, alojada entre la cara lateral y el piso de la órbita. Esta fisura tiene una dirección oblicua (antero-lateral) desde su origen posteromedial a nivel del pilar maxilar (maxillary strut) hasta el hueso cigomático localizado en la región anterolateral del macizo facial. La FOI está relacionada con otras regiones como la órbita, la fosa pterigopalatina (FPtP), la fosa infratemporal (FIT) y la fosa temporal (FT).

De esta manera, y con más detalles, podemos decir que la FOl está delimitada por los siguientes huesos:

$$
\begin{aligned}
& \text {-posteriormente por el ala mayor del } \\
& \text { esfenoide, } \\
& \text {-lateralmente por el hueso cigomático, } \\
& \text {-medialmente por el cuerpo del esfenoide, } \\
& \text {-anteriormente, por el hueso maxilar y un } \\
& \text { pequeño segmento del hueso palatino }
\end{aligned}
$$

El canal o foramen óptico mide aproximadamente $10 \mathrm{~mm}$ de longitud. Lateralmente se separa de la fisura orbital superior (FOS) por un puente óseo llamado pilar óptico. Las paredes están formadas por el cuerpo del esfenoide y el ala menor del esfenoide. La pared lateral del canal óptico está formada por el pilar óptico que se continúa supero lateralmente en clinoide anterior. La FOI tiene contigüidad con la FOS a nivel del extremo medial del segmento posteromedial. La FOS está separada del foramen redondo mayor por otro puente óseo llamado pilar maxilar.

Desde el punto de vista morfométrico, en todos los casos hemos observado que a nivel general la FOI mantiene el mismo aspecto macroscópico: un segmento posteromedial, uno medio y el anterolateral (Ver figura $\mathrm{N}^{\circ} 1$ ).

- El posteromedial, es el más largo de los tres segmentos y es levemente más ancho en su origen para luego afinarse a expensas de su borde anterior formado por la cara posterior del seno maxilar. Se relaciona con el ápex orbitario, fosa pterigopalatina (FPtP) el foramen redondo mayor.

- El segmento medio está representado por el ancho del surco o canal orbitario inferior el cual genera una depresión en el borde anterior de la FOI. En la unión del surco con la FOI observamos disparidad entre ambos bordes del surco: mientras el borde proximal es oblicuo, el distal es perpendicular. Este segmento está relacionado por debajo con la fosa infratemporal (FIT).

- El segmento anterolateral es el más ancho de los tres en términos generales, pero hay casos en los cuales puede ser tan angosto como los otros segmentos, es decir que su ancho presenta una mayor variabilidad. Como se puede apreciar en la figura $\mathrm{N}^{\circ} 3$ presenta dos porciones, una proximal que tiene el mismo ancho que el segmento medio para luego ensancharse en la porción distal. Está relacionado con la fosa 
temporal. La fisura pterigomaxilar comunica esta fosa con la FPtP.

El segmento de la fisura orbitaria inferior que se relacionó con el foramen o canal óptico y con el foramen redondo fue el posteromedial. El foramen óptico se encuentra superior medial a este segmento de la FOI. La fisura orbitaria superior está a su vez separada del foramen redondo mayor por otro septo óseo llamado pilar maxilar el cual es origen de la FOI, más precisamente, de su segmento posteromedial. Además el extremo medial o profundo de este segmento de la FOI converge con la FOS a nivel de la zona de transición entre la región selar y la órbita, llamado ápex orbitario. Esta región es denominada en este trabajo como Junción orbitoselar medial.

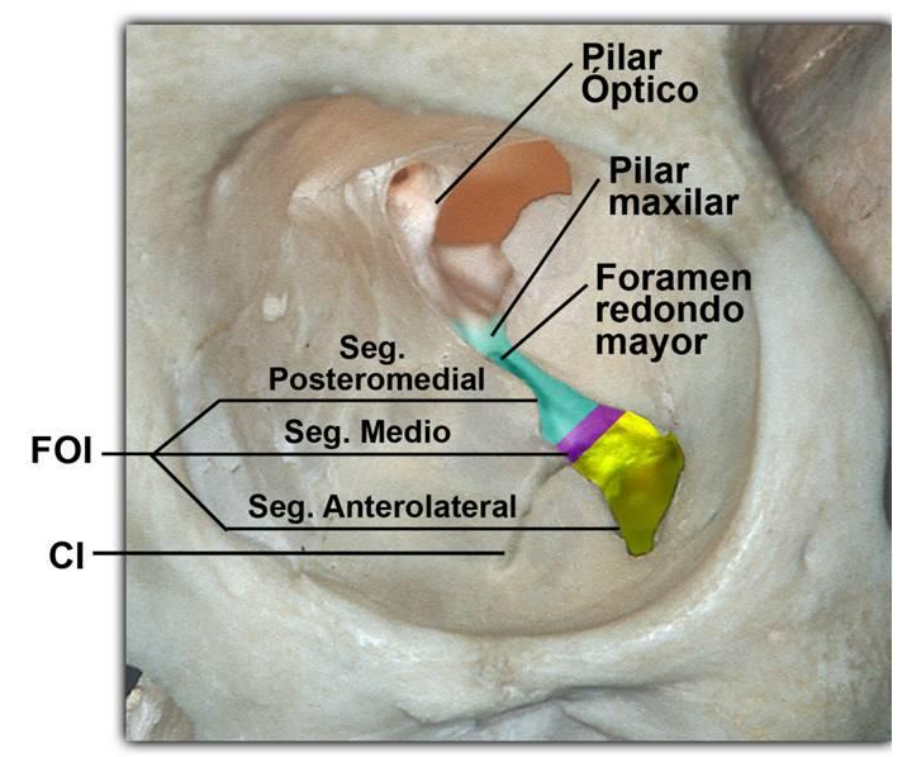

Figura $N^{\circ}{ }^{1}$. Foto de cráneo seco de órbita. Izquierda: muestra los diferentes segmentos de la Fisura Orbital Inferior: 1) Posteromedial (celeste turquesa) que va desde el pilar maxilar al borde posterior del surco o canal orbitario inferior. 2) Medio (Violeta): representado por el canal infraorbitario. 3)

Anterlateral (amarillo): desde el borde anterior del surco o canal infraorbitario hasta el aspecto más anterolateral de la FOI. SIGLAS (CI: Canal o surco Infraorbitario; FOI: Fisura Orbitaria Inferior; Seg.: Segmento).

3-Abordajes quirúrgicos endoscópicos: Se realizó un abordaje endoscópico endonasal amplio en pasos de exposición anatómica progresiva, a mencionar:

Primero, turbinectomía bilateral con septostomía amplia y exposición del seno esfenoidal (esfenoidectomía) y del seno maxilar en cuestión (antrostomía medial endoscópica). Posteriormente resección de la pared medial de la órbita (lámina papirácea) de próxima a distal con fresado de la apófisis orbitaria del palatino y de la cara medial del foramen óptico.

Luego de la disección perióstica - dural de la base del cráneo, se abrió la duramadre de toda la región. Se identificaron los reparos anatómicos endoscópicos clásicos la región selar-hipofisaria. Una vez expuesto el ápex orbitario se mostró el complejo fisura orbitaria inferior-músculo de Müller (FOI-MM) permitiendo ampliar la disección hasta la región del ápex orbitario o junción orbitoselar medial (JOSM) (Figura $\mathrm{N}^{\circ} 2$ ).

Posteriormente, se procedió a la apertura progresiva del anillo de Zinn con la visualización de los diferentes nervios que atraviesan la fisura orbitaria superior (FOS) en dirección a la órbita. Se observó en todos los especímenes una clara y constante relación entre el complejo FOI-MM y la FOS-ápex orbitario (Figura $\mathrm{N}^{\circ} 3$ ). Finalmente se realizó una visión endoscópica transmaxilar directa de esta región.

\section{FISURA ORBITARIA INFERIOR (FOI) $Y$ LA CIRUGÍA ENDOSCÓPICA}

La FOI se encuentra entre la pared lateral e inferior de la órbita y se accede endoscópicamente mediante la apertura de la pared posterior del seno maxilar. Los segmentos de la misma permiten establecer corredores quirúrgicos específicos a las diferentes aéreas contiguas a la FOI, según mostramos en otra publicación enmarcada en nuestra tesis ${ }^{(14)}$.

Cuando utilizamos una vía endoscópica endonasal con apertura del seno maxilar (antrostomía), el segmento posteromedial es lo primero en ser visualizado. Este segmento está en íntima relación con las estructuras encontradas a nivel del ápex orbitario, a saber: 1cara anterior del seno cavernoso, 2- fisura orbitaria superior y 3- orifico redondo mayor y los contenidos de cada una de estas regiones. EI segmento medio también puede ser expuesto por esta vía endoscópica, aumentado la resección ósea. Para lograr una libertad de movimiento del endoscopio necesaria en la resección de tumores es aconsejable, en algunos pacientes, la resección parcial del cornete inferior (cola o tercio medio-posterior). La disección de la pared medial del seno cavernoso y apertura del anulus de Zinn o tendón anular común, nos permitió ver las estructuras neuro-vasculares de la unión orbital selar por su cara medial (Figura $\mathrm{N}^{\circ} 3$ ).

El extremo posterior de la fisura orbitaria inferior (FOI) cruza la porción medial de la fisura orbitaria superior (FOS) justo encima del pilar maxilar. La unión entre el FOS y la FOI estuvo en relación con la inserción del músculo de Müller (MM) a nivel de la confluencia anterior del seno cavernoso (CS) (Figura №3). Este músculo se extiende a lo largo de toda FOI permitiendo el análisis endoscópico de la misma y el estudio de la relación de este músculo con la FOS a nivel del anillo de Zinn. El músculo de Müller presenta 
una íntima relación con el anillo de Zinn, sin una verdadera adherencia del músculo al mismo.

Sin embargo, las llamadas fascias periósticas que recubren a estas dos estructuras, en realidad son una sola, y por lo tanto, indivisibles. Observamos también que la grasa que acompaña o sigue el trayecto de la FOI entra en contacto con la grasa del seno cavernoso (SC). Esto genera que estos diferentes compartimientos 0 áreas estén relacionados entre el exo y el endocráneo, (fosas de la base de cráneo-pterigopalatina e infratemporal, senos cavernosos, órbita), siendo por lo tanto posibles corredores de propagación de los procesos patológicos.

De esta forma el binomio o unidad FOI-MM representa un reparo anatómico endoscópico constante y fiable para el ápex orbitario. La seguridad que brinda esta unidad es doble: por un lado nos permite un reparo óseo, duro (labios de la FOI), y por el otro, uno visible (MM). Este músculo nos marca, además, la zona de fresado seguro del ala mayor del esfenoides (safe zone) para exponer la fosa media, cara lateral del seno cavernoso y ápex del seno cavernoso (abordaje trans-fisura orbitaria inferior).

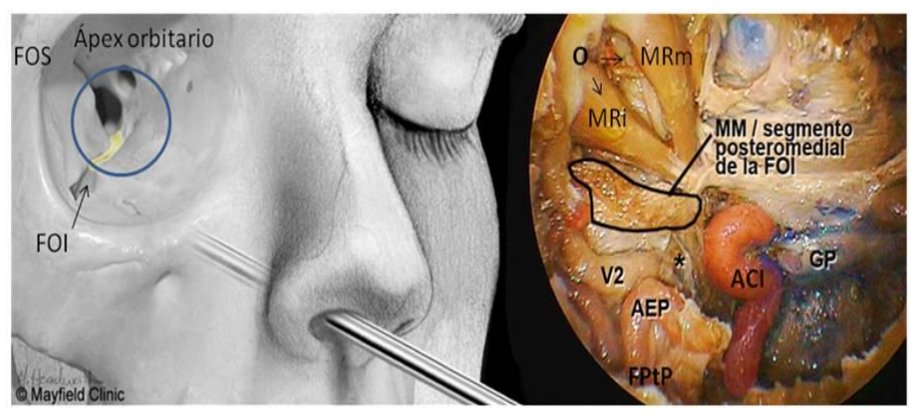

Figura N²: Dibujo izquierdo: muestra la dirección de trabajo en endoscopio (Endoscópico endonasal con máxilo-antrostomía más etmoidectomía parcial). Se puede observar a nivel del ápex orbitario (círculo azul) la unión de la FOI con la FOS y su estrecha relación con el foramen óptico. Foto derecha: Visión endoscópica de la región del ápex orbitario y su correlación con el segmento posteromedial de la FOl. Obsérvese la relación de este segmento con la otra área contigua, la fosa pterigopalatina (FPtP). El nervio maxilar superior (V2) sale del cráneo por el orificio redondo mayor para ingresar a: la fosa FPtP inmediantamente por debajo de la FOI y el MM. Este nervio tiene una trayecto paralelo al segmento posteromedial de la FOI. El asterisco marca el pilar maxilar, techo del foramen redondo mayor, septo óseo, que lo separa de la fisura orbitaria superior. SIGLAS (MM: Músculo de Müller; GP: duramadre que recubre la glándula pituitaria; V2: Nervio Maxilar Superior; AEP: Arteria Esfeno Palatina; FPtP: Fosa Pterigo Palatina; asterisco: Pilar maxilar)

La trayectoria de los nervios que atraviesan la FOS es sumamente compleja y en la visión endoscópica 2D, la complejidad aumenta haciéndose más difícil su reconocimiento. La unidad FOI-MM nos permite conocer el momento de apertura de la FOS con el consiguiente cuidado de las estructuras que la atraviesan (Figura $\mathrm{N}^{\circ} 3$ ). Los nervios que atraviesan la FOS son:

El III par craneal (nervio oculomotor común) el cual viaja por debajo del proceso clinoide anterior y se divide en rama superior e inferior. Estas divisiones entran en el cono muscular ápex orbitario a través del agujero anular superolateral del anillo de Zinn. La rama superior se incorpora rápidamente en el músculo recto superior. La rama inferior avanza y envía una rama pequeña al ganglio ciliar para luego dar tres más que inervan el recto medial o interno, el recto inferior y el músculo oblicuo inferior. El VI y el V1 cruzan por debajo del III para alcanzar el aspecto superolateral del ápex orbitario.

El IV par (nervio troclear o patético) y las ramas frontales de V1 entran en la órbita por encima del origen del músculo recto superior y el foramen anular superolateral. A nivel de la rodilla anterior de la $\mathrm{ACl}$ (arteria carótida interna), el nervio troclear está situado lateral y debajo del nervio oculomotor. El nervio troclear atraviesa progresivamente el músculo recto superior y el músculo elevador del parpado hacia el lado superolateral del músculo oblicuo superior donde lo inerva.

El VI par (Nervio motor ocular externo), su trayectoria ascendente (30 grados aproximadamente) y medial al nervio oftálmico o V1, va desde el canal de Dorello hasta la órbita, terminando en el músculo recto lateral.

La división oftálmica del nervio trigémino (V1) se divide rápidamente a nivel de la rodilla anterior de la $\mathrm{ACI}$ (arteria carótida interna), antes de ingresar a la FOS, en los nervios frontal, nasocilial y lagrimal. Este nervio, desde su nacimiento a nivel del ganglio trigeminal, se sitúa en la parte inferior del SC y lateral al VI par craneal.

Las estructuras relacionadas con el canal o foramen óptico son: El nervio óptico y la arteria oftálmica entran en la órbita a través del agujero anular superomedial del anillo de zinn. La arteria se encuentra en relación a la cara inferior del NO e ingresa dentro de la vaina del mismo. El Anillo Tendinoso de Zinn está unido en los márgenes superior, mediano y inferior del canal óptico (12 a 6 horas). Este tendón se forma a partir de la inserción de los músculos extraoculares: el recto superior, medial, inferior y lateral. 


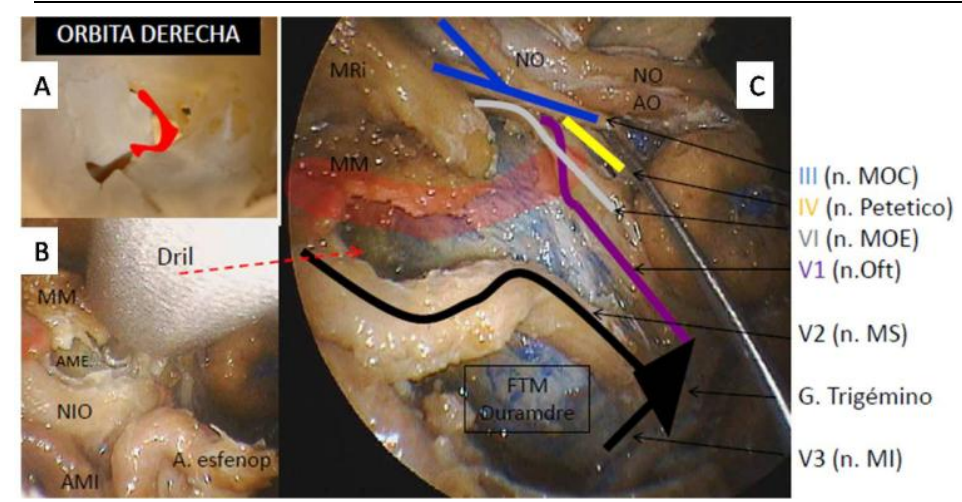

FIGURA Nํ․ Esquema de 3 fotos que muestran la unidad FOI-MM (segmento posteromedial) como reparo anatómico de la junción o unión órbito selar medial. Foto superior izquierda (A): Vista de órbita derecha de un cráneo seco. Se pintó de rojo la FOI y la FOS marcando claramente su relación o unión a nivel del ápex orbitario (junción orbito-selar medial). Foto inferior izquierda (B): El músculo de Müller en la FOI es punto de reparo endoscópico para el fresado del ala mayor del esfenoide: Corredor Trans-fisura orbitaria inferior (safe zone). La flecha punteada indica la zona de comienzo del fresado con el dril. Foto inferior izquierda (Principal o C). Visión endoscópica endonasal derecha. Se realizó apertura de los tendones del anillo de Zinn y resección del músculo de Müller con el objetivo de exponer la junción orbitoselar medial. Se realizó un fresado del parcial del ala mayor del esfenoide (Corredor trans-FOI/MM) y del piso de la fosa media (corredor

transpterigoideo). Se observan los pares craneales que salen de la fisura orbitaria superior para entrar en la órbita. También se observa la entrada de la arteria oftálmica y nervio óptico por el foramen óptico que está separado de la FOS por el pilar óptico llamado endoscópicamente receso-optocarotideo. La FOS está en contacto con la FOI en su extremo posteromedial. Es a este nivel que el músculo de Müller entra en íntima relación con las venas oftálmicas (complejo venoso inferior) que desembocan en el seno cavernoso. La exposición de la anatomía de esta región muestra la relación entre la FOI-FOS-FRM y el ápex orbitario. La unidad FOI-MM es un reparo útil y seguro para la exposición de estas regiones ("safe zone"). SIGLAS (MRI: Músculo recto inferior; MM: Músculo de Müller; NO: nervio óptico; AO: arteria oftálmica; FTM: duramadre de la fosa temporal media; AMI, Arteria maxilar interna, A. Esfenop.: arteria esfenopalatina, NIO: nervio infraorbitario, III: nervio motor ocular común (n.MOC), IV: nervio patético, VI: nervio oftálmico, V2: nervio maxilar superior, G. trigémino: Ganglio trigémino, V3: nervio maxilar inferior.)

\section{Discusión}

El ápex orbitario y sobre todo su cara inferomedial es una región difícil para el acceso neuroquirúrgico abierto. Tradicionalmente se han utilizado abordajes extensos anteriores (transfaciales o transmandibulares) y laterales / posterolaterales (transcraneales) para acceder al mismo. Se han observado frecuentes morbilidades relacionadas con estos abordajes, particularmente de los nervios craneales $^{(21,22,23,24,25)}$. A diferencia de estos abordajes clásicos de la neurocirugía, los abordajes endoscópicos endonasales 0 transmaxilares generan una trayectoria de trabajo directa al target (ápex orbitario) con mayor amplitud de visualización de la unión órbito-selar medial $^{(10,11)}$. A pesar de estas ventajas, la compleja anatomía de la cara ventral de la base de cráneo, y en particular la anatomía endoscópica del ápex orbitario, necesitan sumar puntos de referencia anatómicos endoscópicos intraoperatorios seguros, constantes y analizables, previos a la cirugía y durante la intervención ${ }^{(26,27,28)}$. Cabe señalar, que si bien desde la década pasada se cuenta con una gran serie de estudios en cadáveres sobre la anatomía endoscópica del ápex orbitario, fosa infratemporal (FIT) y fosa pterigopalatina (FPtP), con descripciones detalladas de puntos o reparos anatómicos, ninguno tuvo en cuenta ni se señaló la unidad estructural FOI-MM. $]^{(1,29,30,31)}$. Según nuestra indagación, estos estudios pasaron por alto su descripción, relaciones y la utilidad de este binomio o unidad estructural como un importante reparo anatómico para la FOS, ápex orbitario, FIT y la FPtP. Como describieron Cavallo et al. $^{(30)}$ y Alfieri et al $^{(29)}$, el abordaje endoscópico ofrece una excelente visión del seno maxilar medial y de la fosa pterigopalatina, ápex orbitario y por ende a la FOI y al MM, como mostramos en este artículo. De esta forma nosotros observamos que la unidad o binomio fisura orbitaria inferior-músculo de Müller (FOI$\mathrm{MM}$ ) sirve como reparo anatómico en los abordajes endoscópicos a la región del ápex orbitario o junción órbito-selar medial (JOSM) ya que el mismo llega hasta la cara inferior del seno cavernoso, región inferior de la fisura orbitaria superior y cara medial de ápex orbitario. La exposición de anulus o anillo de Zinn confirmó la relación íntima que presenta con el músculo de Müller, estando unidos a escasos milímetros por la fascia periostica-dural.

Para finalizar señalamos que la observación de la unidad FOI-MM en su segmento posteromedial nos permitió definir, por un lado, márgenes seguros de disección endoscópica en esta región medial órbito-selar con apertura de la FOI, FRM, FOS y el contenido intraobirtario de su ápex; y por otro lado, vimos que genera una zona segura de fresado-exposición de la cara anterior de la fosa media, lateral y anterior del seno cavernoso. Es por esta razón que denominamos a esta región safe zone.

\section{Conclusión}

La unidad Fisura Orbitaria Inferior-Músculo de Müller es un reparo constante, útil y seguro para el reconocimiento del ápex orbitario y sus áreas contiguas, tales como la fisura orbitaria superior, la región anterior e inferior del seno cavernoso y la posterior de la órbita. Por tanto concluimos que el conocimiento anatómico preciso de la relación FOI y el MM posibilita una reconstrucción anatómica visual y conceptual clara de la unión 
FOI-FOS a nivel de lo que denominamos la junción órbito-selar medial. Además, el reconocimiento de esta unidad funcional permite delimitar una zona de seguridad (safe zone) para el fresado y exposición de esta región, el corredor trans-FOI/MM.

\section{Agradecimientos}

Si bien esta mención omite a varias personas que en diferentes momentos nos han apoyado para realizar esta publicación, no queremos dejar de agradecer los valiosos aportes de los Drs. Profesor Domingo Balderramos y Profesor Gustavo Ortiz, Virginia L. Sabattini como también los de Martha E. Headworth.

\section{Bibliografía}

1. Abuzayed B, Tanriover N, Gazioglu N, Eraslan BS, Akar Z. Endoscopic endonasal approach to the orbital apex and medial orbital wall: anatomic study and clinical applications. $J$ Craniofac Surg 2009; 20: 1594-1600.

2. Tsirbas A, Kazim M, Close L. Endoscopic approach to orbital apex lesions. Ophthal Plast Reconstr Surg 2005; 21: 271 275.

3. Tepedino MS, Pinheiro-Neto CD, Bezerra TF, Gardner PA, Snyderman $\mathrm{CH}$, Voegels RL. Endonasal identification of the orbital apex. Laryngoscope. 2016 Jan; 126(1):33-8.

4. Rangel-Castilla L, Russin JJ, Spetzler RF. Surgical management of skull base tumors. Rep Pract Oncol Radiother. 2016 Jul-Aug;21(4):325-35.

5. Kawahara N, Sasaki T, Asakage T, Nakao K, Sugasawa M, Asato H, Koshima I, Saito N. Long-term outcome following radical temporal bone resection for lateral skull base malignancies: a neurosurgical perspective. J Neurosurg. 2008 Mar;108(3):501-10.

6. van Furth WR, Agur AM, Woolridge N, Cusimano MD. The orbitozygomatic approach. Neurosurgery. 2006 Feb;58(1 Suppl).

7. Lemole GM Jr, Henn JS, Zabramski JM, Spetzler RF. Modifications to the orbitozygomatic approach. Technical note. J Neurosurg. 2003 Nov;99(5):924-30. Review.

8. Boari N, Spina A, Giudice L, Gorgoni F, Bailo M, Mortini P. Fronto-orbitozygomatic approach: functional and cosmetic outcomes in a series of 169 patients. J Neurosurg. 2017 Feb 3:1-9.

9. Dziedzic TA, Anand VK, Schwartz TH. Endoscopic endonasal approach to the lateral orbital apex: case report. J Neurosurg Pediatr. 2015 Sep;16(3):305-8.

10. Alimohamadi $M$, Hajiabadi $M$, Gerganov V, Fahlbusch $R$, Samii M. Combined endonasal and sublabial endoscopic transmaxillary approach to the pterygopalatine fossa and orbital apex. Acta Neurochir (Wien). 2015 Jun;157(6):91929; discussion 929. Erratum in: Acta Neurochir (Wien). 2016 Jun;158(6):1229.

11. Elhadi AM, Almefty KK, Mendes GA, Kalani MY, Nakaji $P$, Dru A, Preul MC, Little AS. Comparison of surgical freedom and area of exposure in three endoscopic transmaxillary approaches to the anterolateral cranial base. J Neurol Surg B Skull Base. 2014 Oct;75(5):346-53.

12. Karaki M, Kobayashi R, Mori N. Removal of an orbital apex hemangioma using an endoscopic transethmoidal approach: technical note. Neurosurgery. 2006 Jul;59(1 Suppl 1): ONSE159-60; discussion ONSE159-60.

13. Elhadi AM, Zaidi HA, Yagmurlu K, Ahmed S, Rhoton AL Jr, Nakaji $P$, Preul MC, Little AS. Infraorbital nerve: a surgically relevant landmark for the pterygopalatine fossa, cavernous sinus, and anterolateral skull base in endoscopic transmaxillary approaches. J Neurosurg. 2016 Dec;125(6):1460-1468.

14. De Battista JC, Zimmer LA, Theodosopoulos PV, Froelich SC, Keller JT. Anatomy of the inferior orbital fissure: implications for endoscopic cranial base surgery. J Neurol Surg B Skull Base. 2012 Apr;73(2):132-8. doi:10.1055/s0032-1301398. PubMed PMID: 23542710; PubMed Central PMCID: PMC3424630.

15: De Battista JC, Zimmer LA, Rodríguez-Vázquez JF, Froelich SC, Theodosopoulos PV, DePowell JJ, Keller JT. Muller's muscle, no longer vestigial in endoscopic surgery. World Neurosurg. 2011 Sep-Oct;76(3-4):342-6.

16: Cebula H, Lahlou A, De Battista JC, Debry C, Froelich S. [Endoscopic approaches to the orbit]. Neurochirurgie. 2010 Apr-Jun;56(2-3):230-5.

17. Dallan I, Di Somma A, Prats-Galino A, Solari D, Alobid I, Turri-Zanoni M, Fiacchini G, Castelnuovo P, Catapano G, de Notaris $M$. Endoscopic transorbital route to the cavernous sinus through the meningo-orbital band: a descriptive anatomical study. J Neurosurg. 2016 Nov 18:18.

18. Ulutas M, Boyacı S, Akakın A, Kılıç T, Aksoy K. Surgical anatomy of the cavernous sinus, superior orbital fissure, and orbital apex via a lateral orbitotomy approach: a cadaveric anatomical study. Acta Neurochir (Wien). 2016 Nov;158(11):2135-2148.

19. Natori $Y$, Rhoton AL Jr. Microsurgical anatomy of the superior orbital fissure. Neurosurgery. 1995 Apr;36(4):76275.

20. Natori $Y$, Rhoton AL Jr. Transcranial approach to the orbit: microsurgical anatomy. J Neurosurg. 1994 Jul;81(1):78-86.

21. Fisch $U$, Fagan $P$, Valavanis $A$. The infratemporal fossa approach for the lateral skull base. (1984) Otolaryngol ClinN Am17(3):513-552.

22. Fisch U (1984) Infratemporal fossa approach for lesions in the temporal bone and base of the skull. Adv Otorhinolaryngol 34: 254-266.

23. Hitotsumatsu T, Rhoton AL Jr Unilateral upper and lower subtotalmaxillectomy approaches to the cranial base:microsurgical anatomy. (2000) Neurosurgery 46(6):1416-52.

24. Niho $S$ (1961) Decompression of the optic canal by the transethmoidal route. Am J Ophthalmol 51:659-665.

25. Zhang M, Garvis W, Linder T, Fisch U (1998) Update on the infratemporal fossa.

26. Aust MR, McCaffrey TV, Atkinson J: Transnasal endoscopic approach to the sella turcica. Am J Rhinol 12:283$287,1998$.

27. Kassam AB, Gardner PA, Snyderman CH, Carrau RL, Mintz $A H$, Prevedello DM: Expanded endonasal approach, a fully endoscopic transnasal approach for the resection of midline suprasellar craniopharyngiomas: a new classification based on the infundibulum. J Neurosurg 108:715-728, 2008

28. Kassam AB, Prevedello DM, Carrau RL, Snyderman $C H$, Thomas A, Gardner P, et al: Endoscopic endonasal skull base surgery: analysis of complications in the authors' initial 800 patients. J Neurosurg 114:1544-1568, 2011.

29. Alfieri $A$, Jho $H D$, Schettino R, Tschabitscher $M$ (2003) Endoscopic endonasal approach to the pterygopalatine fossa: anatomic study. Neurosurgery 52(2):374-78.

30. Cavallo LM, Messina A, Gardner P, Esposito F, Kassam AB, Cappabianca $P$, de Divitiis E, Tschabitscher M (2005) Extended endoscopic endonasal approach to the pterygopalatine fossa: anatomical study and clinical considerations. Neurosurg Focus 19(1): E5.

31. Solari D, Magro F, Cappabianca $P$, Cavallo LM, Samii $A$, Esposito F, PaternòV,DeDivitiis E, SamiiM (2007) Anatomical study of the pterygopalatine fossa using an endoscopic endonasal approach: spatial relations and distances between surgical landmarks. J Neurosurg 106(1):157-63. 